\title{
Case Study: the Role of Physical Therapy in the Treatment of Chronic Pelvic Pain with Sexual Dysfunction
}

\author{
Alfarra, Najwa Abdelrahman
}

\begin{abstract}
Background: At King Faisal Specialist Hospital \& RC. The percentage of female chronic pelvic pain increased between all women's who visited the Ob/Gyn according to the departmental monthly report.Clinic. The good news is that physicians and patients have become more aware of the important role that PT plays in the diagnosis and treatment of female chronic pelvic pain.

Study Design: the case study was used to high light the role of physical therapy in the treatment of chronic pelvic pain and sexual dysfunction.

Case Description: 45 years old Saudi female had been diagnosed with right hip pain (bursitis) and pain during intercourse referred to physical therapy department, and given Mobic 15mg. once per day and Voltaren cream to use it at bed time for the last two months, but no improvement on the long run. The physical therapy finding was: minimal to moderate restrictions of the connective tissues and trigger points at the right side of suprapubic, medial, lateral thigh, buttock, iliotibial band, tissue close to vaginal opening and lower back. Minimal difficulty to bear down the pelvic floor and pain with intercourse. Which led to decrease the activity of the sexual life and unable to do the aerobic exercise as a part of her routine life three times per week.

Management and outcomes: The patient received two time per week physical therapy sessions included: manual therapy, dry needling and home exercise program. At the end of third week the patient reported complete relieve of trigger points on the affected areas, she was able to bear down the pelvic floor normally, and able to have free of pain intercourse.

Discussions: this case study showed that the restrictions in the connective tissue of the muscles surrounding the pelvic will lead to sexual dysfunction and chronic pelvic pain. So the proper physical therapy evaluation and treatment can cure the problem.
\end{abstract}

\section{Introduction}

Chronic pelvic pain is a highly prevalent disease at kingdom of Saudi Arabia with negative impact on the quality of life and sexual activity. Nowadays, the role of physical therapy for such conditions became more familiar and the result of physical therapy is often satisfactory and lead to cure the problem. According to Wurn et al.[4] reported that the manual therapy of physical therapy increase the orgasm and decrease pain during the intercourse. Furthermore, this study [3] )showed that intervention of physical therapy in the treatment of chronic pelvic pain is becoming more necessary both in terms of a more refined diagnosis of the clinical situation and in the design of treatment plan. Lamvu [2] added that the women who are diagnosed with chronic pelvic pain, they experienced modest improvement in pain and depression after they received their physical therapy treatment. Study Design:the case study was used to high light the role of physical therapy in the treatment of chronic pelvic pain and sexual dysfunction.In order to help the other therapist to follow the same treatment program.

\section{Case Description: \\ Patient history}

45 years old Saudi female, arrived to the physical therapy clinic with severe right hip pain radiating to the right side of the pelvic, unable to continue with her aerobic exercise and she was unable to tolerate intercourse, she described the pain as a "discomfort." On top of that, she was "tensing up with anticipation of sex" and since the onset of her symptoms, found that although she tried to relax during sex, she could not have complete relaxation. Because of her pain, she decreased intercourse activity. She was diagnosed in the family medicine clinic with right hip pain, and given Mobic 15mg.once a day, Voltaren cream, and PT referral.The Mobic was successful in relieving the pain for few hours then came back with the same severity, however she continued to have it. Her doctor also advised her to use Voltaren cream on the hip overnight. This also did not change her symptoms.

She said that before the chronic pelvic pain, she had never had any pain or difficulty with sex. In addition, she had no urinary or bowel symptoms. She used to do aerobic classes three times per week.

Obviously, a patient's ultimate goal with PT is to get better! But, as both patient and PT embark upon that goal, it's important to set more specific, attainable goals along the way. This allows both patient and PT to assess progress throughout the course of therapy. 
At the beginning of treatment, I worked with her to set both short-term and long-termgoals. When Ireferred to a patient's "long-term" goals I meant to set achievable goals, and I explained to her that the time it will take to achieve the short term goals will be less than what will be needed to achieve the long-term goals.Initially, for her long-term goals, shewanted to be able to tolerate intercourse withfree of pain and return to the same sexual activity as before. As for her short-term goals, she wanted to see a decrease in pelvic floor muscle tone and trigger points by $90 \%$.

\section{Assessment}

\section{External examination}

Muscle palpation:

Check the connective tissueelasticity for the following muscles:

- Connective tissue of abdomen and suprapubic area: suprapubic: minimum restriction.

- Connective tissue of bony pelvis, labia, perineum area, through groin area, ischial tuberosity (IT), and anterior through suprapubic: moderate restriction.

- Connective tissue medial and lateral thighs right side: moderate restriction.

- Connective tissue buttocks right side: minimum.

- Connective tissue upper thighs right side: moderate restriction closer to IT, and tissue close to vaginal opening.

- Connective tissue low back right side: minimal restriction.

External Muscular Assessment:

- Trigger points located in: superficial transverse perineal/ from external palpation, right piriformis, right gluteusmedius,minims, andmaximus.

- Delayed and incomplete relaxation of pelvic floor muscles.

- Poor quality of concentric contraction.

- Minimal to moderate difficulty bearing down

Visual inspection of Vulva/Vestibule/Pelvic Floor Muscle:

- The perineal reflex and anal wink were normal, could bear down with minimal to moderate difficulty.

Q-Tip Test:

- Normal.

\section{Internal examination}

Internal Pelvic Floor Muscle Assessment

- Tone is increased globally.

- Connective tissue lateral to urethra and urethral sphincter: normal.

- Urogenital diaphragm: bilaterally decreased length.

- Trigger points: located superficial transverse perineal right side.

\section{Findings}

Patient presents with increased pelvic floor muscle tone globally, restricted connective tissue mobility in the above-mentioned structures, and muscular trigger points on her right side of the pelvic girdle and pelvic floor musculature. She also presents with delayed voluntary relaxation.

- These impairments contribute to her symptoms of pelvic pain. I am able to reproduce her pain with connective tissue mobilization to the trigger point of right piriformis, right gluteus medius, minims, and maximus.

In addition, the above impairments contribute to patient's functional limitations of her sexual activity which impacts her ability to have intercourse with her partner.Unable to do the aerobic exercises. She is very anxious regarding her condition, and fear avoidance behaviors that contribute to the physical impairments of shortened pelvic floor muscles and active spasms.

\section{Intervention}

Initial Treatment Plan:

- PT twice a week for 4 weeks: Treatment to include: manual physical therapy techniques to restricted tissues; connective tissue mobilization to gluteus muscles, and piriformis muscles.

- Dry needling for gluteus maximus, medius, and minims, and piriformis muscle.

- Initial home program: deep breathing; and relaxation techniques/pelvic floor drops.

\section{Outcome}

After six visits, I assessed the following changes:

- Patient was able to relax the pelvic floor muscles normally.

- Patient was able to have pain free intercourse.

- Free trigger points on gluteus maximus, medius, and minims, and piriformis muscle. 
- Normal connective tissues around the affected structure.

My assessment:

- Pelvic floor muscle hypertonicity persists, although is lessened;

- Trigger points in gluteus maximus, medius, and minims, and piriformis muscle were disappeared.

- Able to voluntarily relax the pelvic floor muscles after contraction;

New Plan of Care:

- Continue PT once a week for another two weeks

- Continue with manual techniques to the connective tissues in the pelvic floor muscles.

\section{New Goals:}

- Continue to pursue long-term goals

- Transition to light aerobic exercise program with no flare ups of symptoms

After a total of 10 visits (she was able to decrease her number of visits to one every three week at visit number ten), I discharged her with all of her goals met and her symptoms eradicated. After visit number six she had transitioned to intercourse. In addition, she returned to aerobic, and sexual activity with no pain. She stopped using Mobic and Voltaren cream.

\section{Discussion}

Based on my results, I strongly supported the use of dry needling to the trigger points of the muscles and the manual therapy for the restricted tissues which will lead to the relaxation of the muscles. This case study showed that the restrictions in the connective tissue of the muscles surrounding the pelvic will lead to sexual dysfunction and chronic pelvic pain. So the proper physical therapy evaluation and treatment can cure the chronic pelvic pain and sexual dysfunction. Additionally, the physical therapist should pay extra attention during the assessment of muscles around the pelvis in order to help the therapist to find the proper diagnosis and plan of treatment.

\section{Conclusions}

The restrictions in the connective tissue of the muscles around the pelvis can be the primary cause of chronic pelvic pain and sexual dysfunction. The physical therapy different modalities can cure this problem.

\section{References}

[1]. Brain Budgell, Guidelines to the writing of case studies, J Can Chiropr Assoc. 2008, 52 (4):199-204.

[2]. Lamvu G, Williams R, Zolnoun D. Long-term outcomes after surgical and nonsurgical management of chronic pelvic pain: one year after evaluation in a pelvic pain specialty clinic. Am J Obstet Gynecol. 2006; 195(2):591-598.

[3]. Tu FF, Holt J, Gonzales J, Fitzgerald CM. Physical therapy evaluation of patients with chronic pelvic pain: a controlled study. Am J Obstet Gynecol. 2008; 198(3):272.e1-272.e7.

[4]. Wurn LJ, Wurn BF, King CR, Roscow AS, Scharf ES, Shuster JJ. Increasing orgasm and decreasing dyspareunia by a manual physical therapy technique. MedGenMed. 2004; 6(4):47. 